\title{
ANKYLOSING SPONDYLITIS PATIENT WITH ETANERCEPTINDUCED CUTANEOUS LUPUS
}

Fernanda Freire Kosac ${ }^{1, \star}$, Roberto Rodrigues de Souza Filho ${ }^{1}$, Jamille Nascimento Carneiro ${ }^{1}$, André Moreira Lemes ${ }^{1}$, Vanessa Burini Lobo ${ }^{1}$, Luciana Nenes Assis Daameche ${ }^{1}$

1.Instituto Hospital de Base do Distrito Federal, Brasília (DF), Brazil.

*Corresponding author: fernandafkosac@gmail.com

\section{BACKGROUND}

Anti-TNF alpha drugs are effective treatments against some inflammatory diseases. Approximately one in four patients treated with anti-TNF agents develops cutaneous adverse events, typically months to years after the initiation of treatment, with xerosis cutis, eczema, psoriasis, palmoplantar pustulosis, cutaneous infections and alopecia, being the most frequently. Etanercept cutaneous side effects are rare and includes injection site reactions, cutaneous lupus, and cutaneous vasculitis.

\section{CASE REPORT}

We report a case of a 34-year-old patient, in current use of sodium diclofenac, who complained of inflammatory neck and low back pain without irradiation. He was referred to rheumatology for evaluation, with limited cervical rotation. On physical examination, the cervical rotation was restricted to 30 degrees on the right and 35 degrees on the left. The modified Schober test was $4.2 \mathrm{~cm}$. Laboratory tests showed negative for rheumatoid factor, antinuclear factor and HLAB27. There was a slightly increased erythrocyte sedimentation speed and C-reactive protein. Magnetic resonance imaging of sacroiliac showed bilateral ankylosis. The diagnosis of ankylosing spondylitis was made and etanercept $50 \mathrm{mg}$ per week was started, with remission of the back pain. After one year using only etanercept, the patient comes to the consultation complaining of erythematous lesions infiltrating the face and the armpits (Figs. 1 and 2). He returned with the following results: negative for ANA, anti-SM, anti-RO, anti-LA, anti-DNA, anti-RNP, normal complement and positive for anti-histone at $24 \mathrm{UR} / \mathrm{mL}$ (reference value: $<20$ ). At skin biopsy, an interface dermatitis was described, with thickening in foci of the basement membrane area, with a light lymphoplasmacytic infiltrate, suggesting that systemic lupus erythematosus should be evaluated among the possibilities (Fig. 3). It was decided to switch the medication to secukinumab. In the follow-up, the lesions regressed and the disease activity was controlled.

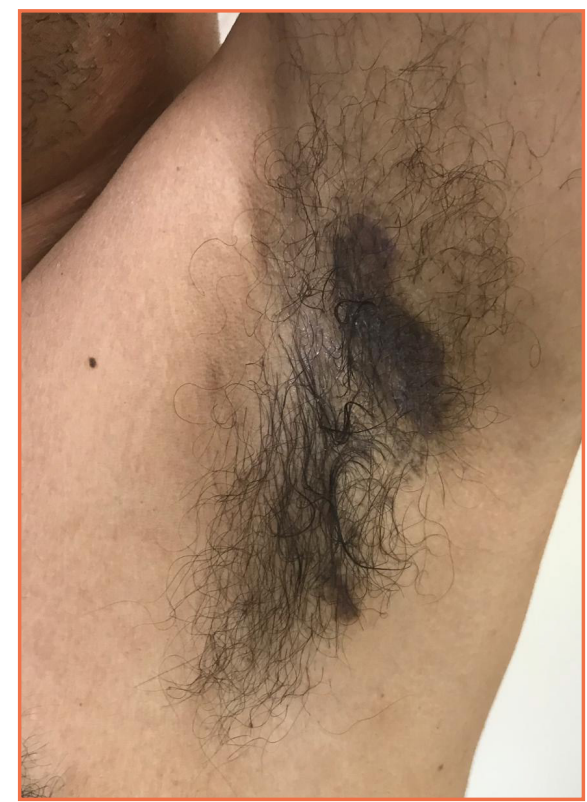

Figure 1. Hyperchromic lesion on the armpit.

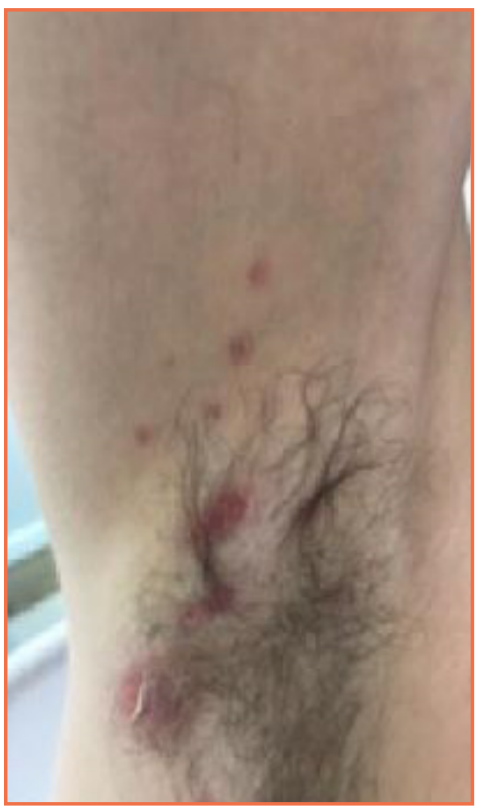

Figure 2. Hyperemic lesions on the armpit. 


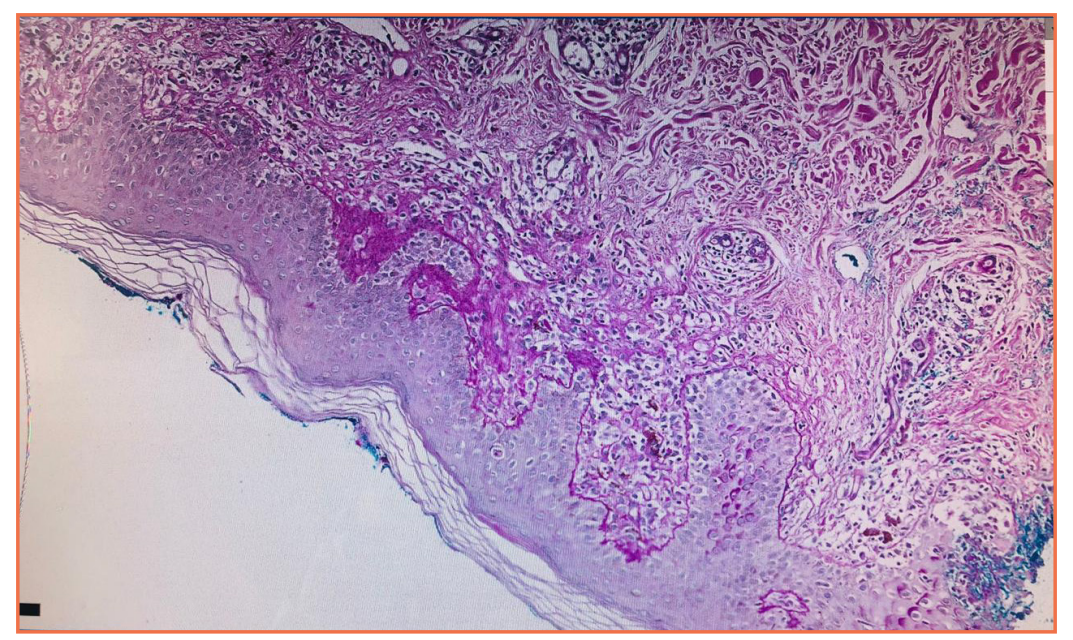

Figure 3. Skin biopsy.

\section{CONCLUSION}

Anti-TNF alpha drugs may induce cutaneous adverse events. Smoking, female sex, and Crohn's disease are risk factors for these events. They can also induce cutaneous lupus. The skin biopsy and the histological analysis with immunofluorescence confirm the diagnosis. Rash is the most common characteristic of lupus induced by anti-TNF agents. In addition to skin lesions, it is known that drug-induced lupus could cause arthritis, hypocomplementemia, hematological abnormalities and the presence of ANA and anti-DNA. Serositis, myositis and neurological disorders are less common. Anti-histone antibodies are found in 75\% of DIL cases. Most of these case reports occurred in patients receiving infliximab or etanercept. By now, comprehensive safety data has been complied. While adverse reactions do occur, they are rare and usually not serious, as we can observe in this case. 